Research Paper

\title{
Combined inhibition of autophagy and Nrf2 signaling augments bortezomib-induced apoptosis by increasing ROS production and ER stress in pancreatic cancer cells
}

\author{
Xu Li1 ${ }^{1,}$, Meng Liang2, ${ }^{2}$, Jianxin Jiang ${ }^{3}$, Ruizhi He${ }^{1}$, Min Wang1, Xingjun Guo ${ }^{1,}$, , Ming Shen ${ }^{1,}$, , Renyi Qin ${ }^{1,}$ \\ 1. Department of Biliary-Pancreatic Surgery, Affiliated Tongji Hospital, Tongji Medical College, Huazhong University of Science and Technology, Wuhan, \\ Hubei, China \\ 2. Department of Hepatobiliary Surgery, Affiliated Hospital of Hebei University, Baoding, Hebei, China \\ 3. Department of Hepatic-Biliary-Pancreatic Surgery, Renmin Hospital of Wuhan University, Wuhan, China. \\ *These authors contributed equally to this work.
}

$\triangle$ Corresponding authors: Renyi Qin, MD, Department of Biliary-Pancreatic Surgery, Affiliated Tongii Hospital, Tongii Medical College, Huazhong University of Science and Technology, 1095 Jiefang Ave, Wuhan, Hubei 430030, China. Tel: +86-27-8366-5294; Fax: +86-27-8366-5294; E-mail: ryqin@tjh.tjmu.edu.cn, Ming Shen, Ph.D, Department of Biliary-Pancreatic Surgery, Affiliated Tongji Hospital, Tongji Medical College, Huazhong University of Science and Technology, Wuhan, Hubei 430030, China. Tel: +86-27-8366-5214; Fax: +86-27-8366-5294 E-mail: shenming-2002@163.com and Xingjun Guo, Ph.D, Department of Biliary-Pancreatic Surgery, Affiliated Tongji Hospital, Tongji Medical College, Huazhong University of Science and Technology, Wuhan, Hubei 430030, China. Tel: +86-27-8366-5294; Fax: +86-27-8366-5294 E-mail: xjguo@tjh.tjmu.edu.cn

(c) Ivyspring International Publisher. This is an open access article distributed under the terms of the Creative Commons Attribution (CC BY-NC) license (https://creativecommons.org/licenses/by-nc/4.0/). See http://ivyspring.com/terms for full terms and conditions.

Received: 2018.04.19; Accepted: 2018.07.02; Published: 2018.07.27

\begin{abstract}
Pancreatic cancer (PC) is highly resistant to current therapies; thus, there is an urgent need to develop new treatment strategies. The proteasome is crucially important for proteostasis, which is involved in cell proliferation and survival, making it an attractive therapeutic target in cancer. However, recent studies have indicated that bortezomib, a highly selective proteasome inhibitor, has limited effects in solid tumors including PC. Thus, more mechanistic insights into chemo-sensitization strategies for bortezomib are urgently needed. Herein, we demonstrate that bortezomib induced apoptosis and autophagy via a mechanism involving endoplasmic reticulum (ER) stress in PC cells. Additionally, bortezomib treatment led to increased levels of intracellular reactive oxygen species (ROS), which play critical roles in bortezomib-induced ER stress and apoptosis. Moreover, autophagy functions as a compensatory mechanism to eliminate bortezomib-induced ROS and resists ER stress-mediated apoptosis. Additionally, the Nrf2-mediated antioxidative response, which works against with bortezomib-induced autophagy, also protected cells against bortezomib-induced ROS production. Finally, the dual inhibition of autophagy and Nrf2 signaling cooperatively enhanced bortezomib-induced apoptosis by elevating ROS levels and ER stress. Together, these data demonstrate that activation of autophagy and the Nrf2 antioxidant system, which lowers intracellular ROS, are mechanistically how PC cells overcome bortezomib treatment. In summary, combining proteasome inhibitors with drugs targeting autophagy and Nrf2 signaling could be a promising therapeutic approach for PC treatment.
\end{abstract}

Key words: Autophagy, Nrf2, Pancreatic cancer, Bortezomib, ROS, ER stress

\section{Introduction}

Pancreatic cancer (PC) is among the most lethal malignant tumors; despite advances in early diagnosis and treatment, its 5-year survival rate is less than $5 \%$ and the median survival is only 6 months [1]. Surgical resection is the only potentially curative treatment but is only appropriate for a minority of patients, as most present with metastatic disease. Unfortunately, approved therapeutic approaches such as radiotherapy and chemotherapy have a relatively modest impact on survival, extending survival by an average of 1-3 months [2]. Thus, there is a continuing need to develop novel therapeutic strategies for PC.

The $26 \mathrm{~S}$ proteasome-mediated degradation of intracellular proteins is highly regulated in eukaryotic cells. Numerous data suggest that the proteasome 
mediates the degradation of proteins involved in cancer cell proliferation, survival and apoptosis, making it an attractive therapeutic target [3]. Bortezomib, a highly selective and potent proteasome inhibitor with broad anti-tumor activities, is actively being investigated as a potential chemotherapeutic agent [4]. It has been reported that the antitumor activity of bortezomib is achieved by affecting various signaling cascades, including the NF- $\mathrm{KB}$, mitogen-activated protein kinases (MAPKs), and apoptotic pathways [5]. Based upon highly favorable results in patients with refractory or relapsed multiple myeloma, bortezomib was approved by the United States Food and Drug Administration [6]. However, recent studies have indicated that single-agent bortezomib has somewhat limited effects in solid tumors including PC, probably due to chemo-resistance or other unknown mechanisms [7, 8]. Thus, more mechanistic insights into chemo-sensitization strategies for bortezomib are urgently needed.

The endoplasmic reticulum (ER) is an organelle that plays important roles in maintaining intracellular calcium homeostasis, protein metabolism and posttranslational modifications. An alteration in calcium homeostasis and/or accumulation of misfolded proteins in the ER results in cellular stress that causes a specialized response known as the unfolded protein response (UPR), which is the major protective and compensatory mechanism for ER stress $[9,10]$. However, if the stress is too severe or persistent, the same system will trigger cell death by inducing pro-apoptotic factors such as C/EBP homologous protein (CHOP) [11]. In most cases, misfolded proteins produced by ER stress are exported to the cytoplasm and degraded by the ER-associated ubiquitin-proteasome degradation (ERAD) system [12]. Nevertheless, if the amount of misfolded proteins exceeds the capacity of the ERAD system, autophagy can compensate for protein degradation and allow cell survival [13].

Autophagy is a lysosomal degradation pathway that eliminates damaged organelles, recycles materials and protein aggregates. Like apoptosis, autophagy is an evolutionarily conserved process that regulates cell fate in response to various stresses [14]. Besides its cytoprotective function, autophagy can also contribute to cell death. However, whether autophagy serves a protective or detrimental role varies depending on cell type and context [15]. Recently, a variety of chemotherapy agents, including bortezomib, were reported to activate autophagy in PC, suggesting that blocking autophagy could enhance its therapeutic efficacy [16, 17]. Thus, a treatment approach combining autophagy inhibition and may reverse the chemo-resistance in PC.

Reactive oxygen species (ROS) production is one of the most important antitumor mechanisms shared by all non-surgical therapeutic approaches, including chemotherapy and radiotherapy [18, 19]. Recent studies have found that a consequence of ER stress is the accumulation of ROS, which promotes oxidative stress [20]. Furthermore, several reports have shown that ROS can induce autophagy in response to chemotherapy-induced stress [21, 22]. In addition to the induction of autophagy, oxidative stress also activates the NF-E2-related factor 2 (Nrf2) pathway. $\mathrm{Nrf} 2$ is a critical regulator of intracellular antioxidants and detoxification enzymes that induces antioxidant response element (ARE)-driven genes expression, such as heme oxygenase-1 (HO-1) and $\mathrm{NAD}(\mathrm{P}) \mathrm{H}$ quinone oxidoreductase 1 (NQO1) [23, 24]. Additionally, aberrant Nrf2 overexpression has been observed in several human cancers, and constitutive Nrf2 activation protects cancer cells against oxidative stress and contributes to the chemo-resistance [25, 26]. However, the molecular link between ROS, ER stress, Nrf2, and autophagy and how these pathways influence therapeutic outcomes in PC remain largely undefined. In this study, we show a novel mechanism of bortezomib resistance and an effective treatment combination to overcome bortezomib resistance.

\section{Results}

\section{Bortezomib inhibits growth and induces apoptosis in PC cells.}

To evaluate the effect of bortezomib on the growth of PC cells, MIAPaCa-2 and AsPC-1 cells were treated with increasing concentrations of bortezomib (1-500 nM) for 24 or $48 \mathrm{~h}$, and then the Cell Counting Kit-8 (CCK-8) assay was performed. As indicated in Fig. 1A, cell growth was inhibited by bortezomib in a dose- and time-dependent manner. Additionally, analyzing long-term survival with colony-formation assays further confirmed that bortezomib inhibited the proliferation of both MIAPaCa-2 and AsPC-1 cells in a dose-dependent manner (Fig. 1B).

To investigate whether the inhibitory effects of bortezomib on PC cells were due to the induction of apoptosis, MIAPaCa-2 and AsPC-1 cells were treated with bortezomib at different concentrations for $24 \mathrm{~h}$, and then the cells were analyzed by Annexin V-FITC/PI staining by flow cytometry. As shown in Fig. 1C, bortezomib treatment increased the percentage of apoptotic MIAPaCa- 2 and AsPC- 1 cells in a dose-dependent manner. These results indicate that bortezomib inhibits growth and induces apoptosis in PC cells. 


\section{Bortezomib activates caspase cascades and the mitochondrial apoptosis pathway in PC cells.}

The mitochondrial apoptosis pathway is one of the critical apoptotic pathways. Depletion of the mitochondrial membrane potential $(\Delta \psi \mathrm{m})$ in response to various stimuli results in the initiation of apoptotic cascades [27, 28]. Therefore, we first investigated if bortezomib-induced apoptosis was associated with the destruction of mitochondrial homeostasis. As indicated in Fig. 1D, bortezomib treatment resulted in a dose-dependent loss of $\Delta \psi \mathrm{m}$ in MIAPaCa-2 and AsPC-1 cells. Additionally, we examined the activation of different caspases and the expression of a
A

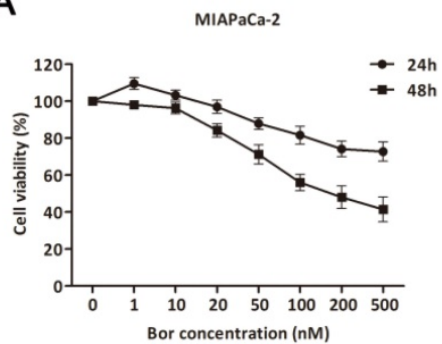

ASPC-1

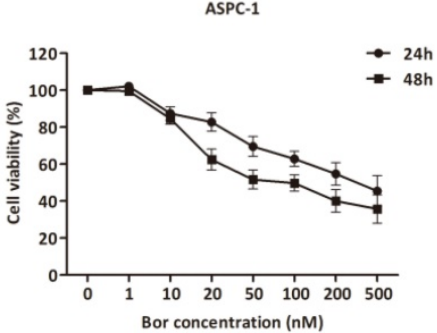

B

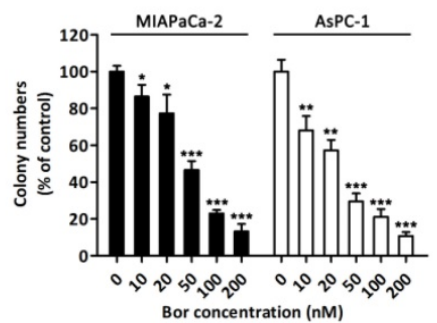

C
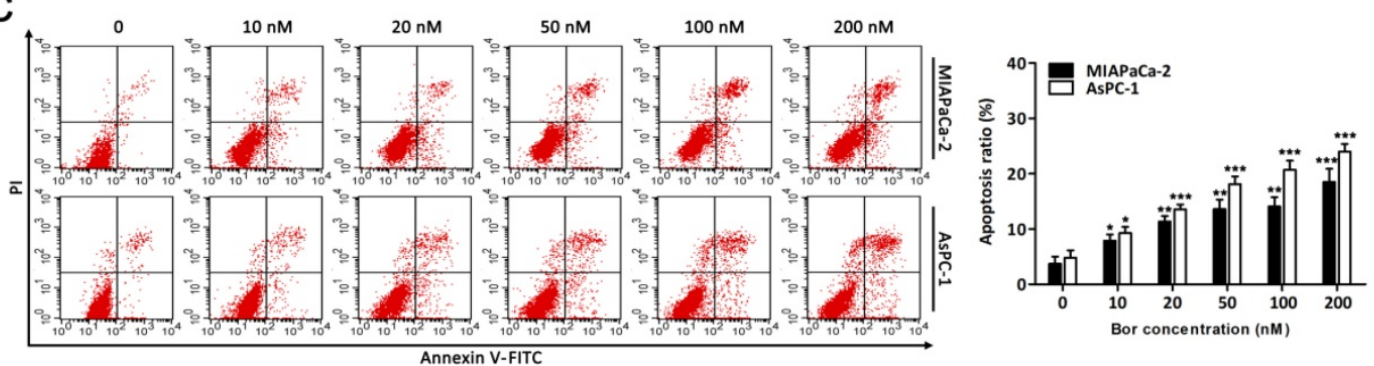

D
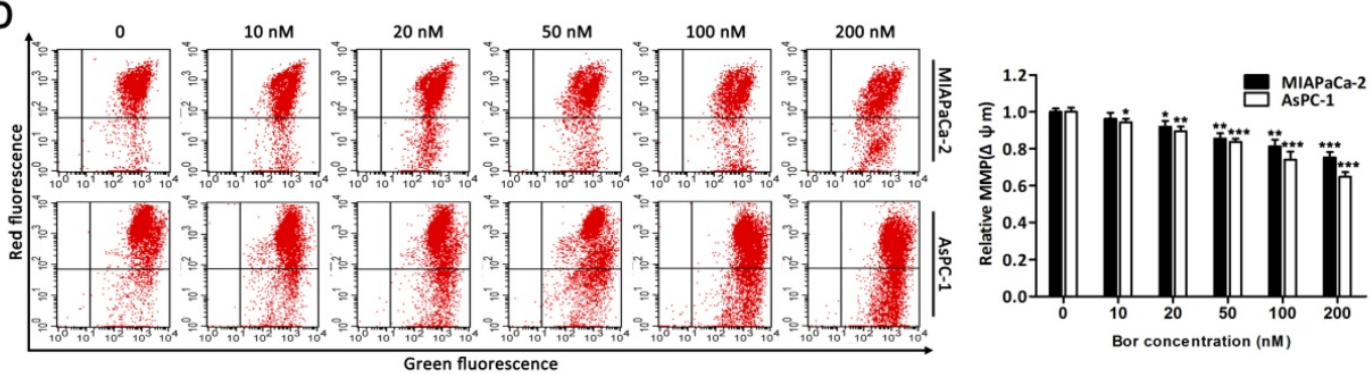

E

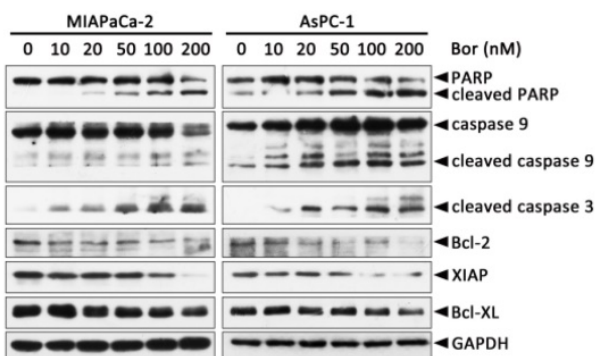

F

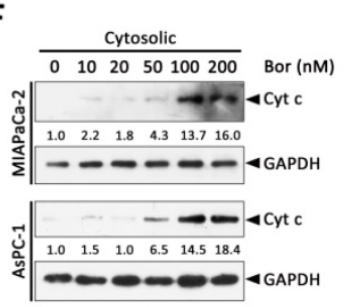

G

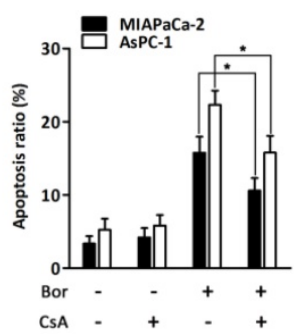

Figure 1. Bortezomib inhibits growth and activates the mitochondrial apoptosis pathway in PC cells. (A) MIAPaCa-2 and AsPC-1 cells were treated with increasing concentrations of bortezomib (1-500 nM) for 24 or $48 \mathrm{~h}$, viable cells were quantified using the CCK-8 assay. Each data point represents means \pm SD of three separate experiments. (B) MIAPaCa-2 and AsPC-1 cells were incubated with the indicated concentrations of bortezomib for $24 \mathrm{~h}$ and allowed to grow into colonies for $14 \mathrm{~d}$. Colony-formation assay was performed to determine the cytotoxic effect of bortezomib. Each bar represents means \pm SD of three separate experiments. ${ }^{*} \mathrm{p}<0.05$; $* * \mathrm{p}<0.01$; $* * * p<0.001$. (C) Apoptotic cells were determined by flow cytometry using annexin V/PI double staining after cells were treated with the indicated concentrations of bortezomib for $24 \mathrm{~h}$. Each bar represents means \pm SD of three separate experiments. *p < 0.05; **p < 0.01; ***p < 0.001. (D) MIAPaCa-2 and AsPC-1 cells were treated as described in (C), and the mitochondrial membrane potential (MMP, $\triangle \psi \mathrm{m}$ ) was determined by JC-1 staining and analyzed by flow cytometry. Each bar represents means \pm SD of three separate experiments. ${ }^{*}<<0.05$; ${ }^{* *} p<0.01 ;{ }^{* * *} p<0.001$. (E) Western blot analysis of PARP, caspase 9 , cleaved caspase-3 and Bcl-2 family proteins levels after cells were treated as described in (C). GAPDH was used as a loading control. (F) Western blot analysis of cytochrome c release from mitochondria after cells were treated as described in (C). GAPDH was used as cytosolic internal control. The band intensity was quantified by volume densitometry and normalized to that of GAPDH. (G) MIAPaCa-2 and AsPC-1 cells were treated with bortezomib $(100 \mathrm{nM})$ for $24 \mathrm{~h}$ in the presence or absence of $\mathrm{CsA}(5 \mu \mathrm{M})$. Apoptotic cells were detected by flow cytometry using annexin V/PI double staining. Each bar represents means \pm SD of three separate experiments. ${ }^{*} \mathrm{p}<0.05$ 
series of Bcl-2 family proteins that play pivotal roles in regulating mitochondrial apoptosis. These results showed that bortezomib dose-dependently induced cleavage of caspase-9, caspase-3 and PARP, and decreased the levels of the anti-apoptotic Bcl-2 family proteins Bcl-2, XIAP, and Bcl-XL in MIAPaCa-2 and AsPC-1 cells (Fig. 1E). Interestingly, pretreating cells with z-VAD-FMK (a pan-caspase inhibitor) significantly attenuated bortezomib-induced cell death (Fig. S1), suggesting that bortezomib-mediated PC cell apoptosis was caspase dependent.

By definition, dissipation of the $\Delta \psi \mathrm{m}$ induces the release of apoptogenic factors such as cytochrome $\mathrm{c}$ from mitochondria to the cytosol [28]. Consistent with this result, bortezomib treatment increased mitochondrial cytochrome c release (Fig. 1F); however, when cells were pre-treated with CsA (a $\Delta \psi \mathrm{m}$ stabilizer), bortezomib-induced cell death was partially blocked (Fig. 1G). These results indicate that bortezomib induces apoptosis by activating a mitochondrial apoptotic pathway.

\section{Bortezomib induces autophagic flux in PC cells.}

As it is well established that proteasome inhibitors also induce autophagy in various cell types [29], we next investigated whether bortezomib induced autophagy in PC cells. First, we monitored autophagic alterations by analyzing the conversion of cytosolic light chain 3B-I (LC3B-I) into LC3B-II. Generally, the covalent conjugation of a soluble form of LC3B (LC3B-I) with phosphatidylethanolamine, forming the insoluble LC3B-II, correlates with the extent of autophagy [14]. As shown in Fig. 2A, bortezomib treatment of MIAPaCa- 2 and AsPC- 1 cells induced a dose-dependent increase in LC3B-II expression. To further monitor autophagosome formation, a lentivirus vector encoding GFP-LC3B fusion protein was used. As expected, bortezomib treatment increased GFP-LC3B puncta in both MIAPaCa-2 and AsPC-1 cells, indicating that there was a cumulative increase in autophagosomes (Fig. 2B). Moreover, ultra-structural analyses by transmission electron microscopy (TEM) revealed increased autophagosome numbers in the cytoplasm of bortezomib-exposed MIAPaCa-2 and AsPC-1 cells (Fig. 2C). We next examined the effects of bortezomib on the expression of several critical autophagy-related proteins. Paradoxically, Beclin1 expression was markedly decreased in bortezomib-treated MIAPaCa-2 cells, whereas no apparent changes were detected in Atg5 or Atg7 expression (Fig. S2).

As both autophagy induction and impaired autophagic degradation have been ascribed to autophagosome accumulation in cells [30], the effect of inhibiting the lysosomal turnover of autophagosome contents by the lysosome inhibitor chloroquine (CQ) was examined. As indicated in Fig. 2D, CQ treatment dramatically increased bortezomibinduced LC3B-II accumulation in both MIAPaCa-2 and AsPC-1 cells, suggesting bortezomib induced autophagy activation. To further confirm that bortezomib induces autophagic flux, a tandem GFP-mRFP-LC3B lentivirus construct was used. This probe can be used to identify autophagosomes (GFP positive/RFP positive; yellow dots) and autolysosomes (GFP-negative/RFP-positive; red dots), because GFP fluorescence is sensitive to $\mathrm{pH}$ and quenched in the acidic lysosomal environment, whereas mRFP is resistant [30]. As shown in Fig. 2E, CQ inhibited autophagosome maturation, resulting in the accumulation of yellow puncta without any significant increase in the number of red puncta in MIAPaCa-2 cells. Conversely, in bortezomib-treated cells, only parts of the LC3B-positive puncta were yellow, indicating increased autophagic flux. Similar results were found using AsPC-1 cells (Fig. S3). Collectively, these data demonstrate that bortezomib induces autophagic flux in PC cells.

\section{Bortezomib-induced apoptosis and autophagy are associated with ER stress.}

It has been hypothesized that proteasome inhibition may prevent the clearance of misfolded proteins by the ERAD pathway, resulting in ER stress [31]. As expected, the level of ubiquitinated proteins in MIAPaCa-2 and AsPC-1 cells were dosedependently increased after bortezomib treatment (Fig. 3A), suggesting bortezomib inhibited proteasome activity. To verify whether ER stress was a consequence of bortezomib-induced proteasome inhibition, cells were stained with the ER-specific marker calnexin. In untreated cells, the ER had a reticular pattern, whereas following bortezomib treatment, numerous cytoplasmic vacuoles appeared (Fig. 3B). Furthermore, bortezomib dose-dependently increased expression of the ER stress-associated proteins Bip and CHOP (Fig. 3C), JNK activation (Fig. 3C) and intracellular calcium levels (Fig. 3D), which are also important markers of ER stress [11], indicating ER stress in bortezomib-treated PC cells.

Emerging evidence has indicated that ER stress is a potent trigger of autophagy [13, 32]; moreover, $\mathrm{CHOP}$ is generally considered a pro-apoptotic factor during ER stress that regularly participates in ER stress-mediated autophagy [33, 34]. Therefore, to further establish the functional role of ER stress in bortezomib-induced PC cell autophagy, CHOPspecific siRNA was used. As shown in Fig. 3E, CHOP-specific siRNA effectively silenced $\mathrm{CHOP}$ at 
A

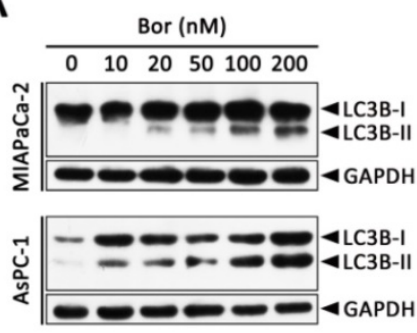

B

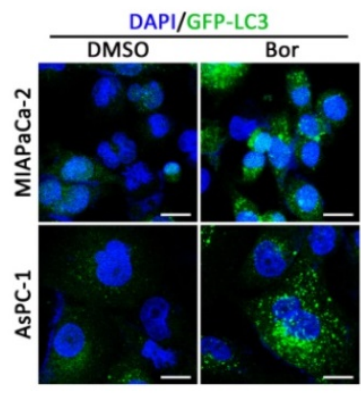

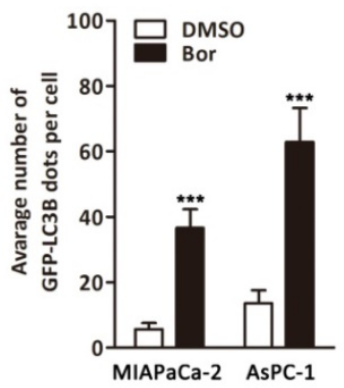

D

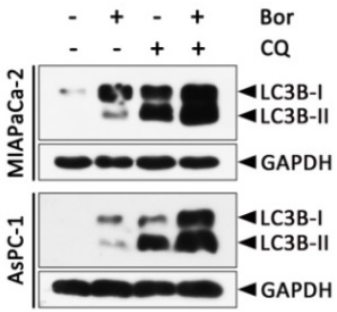

C
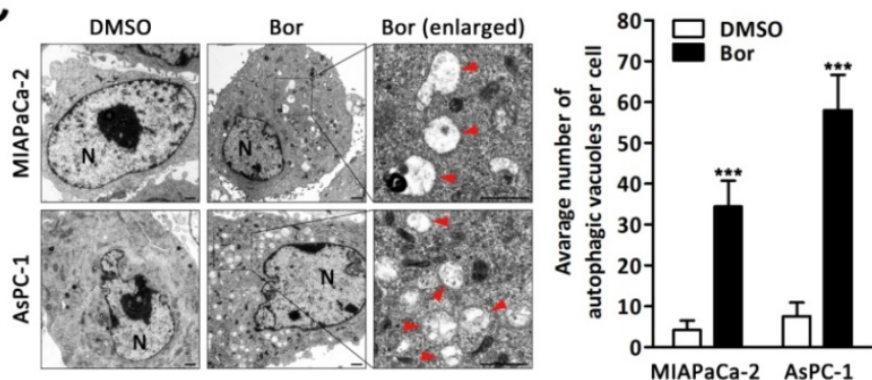

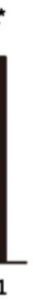

E
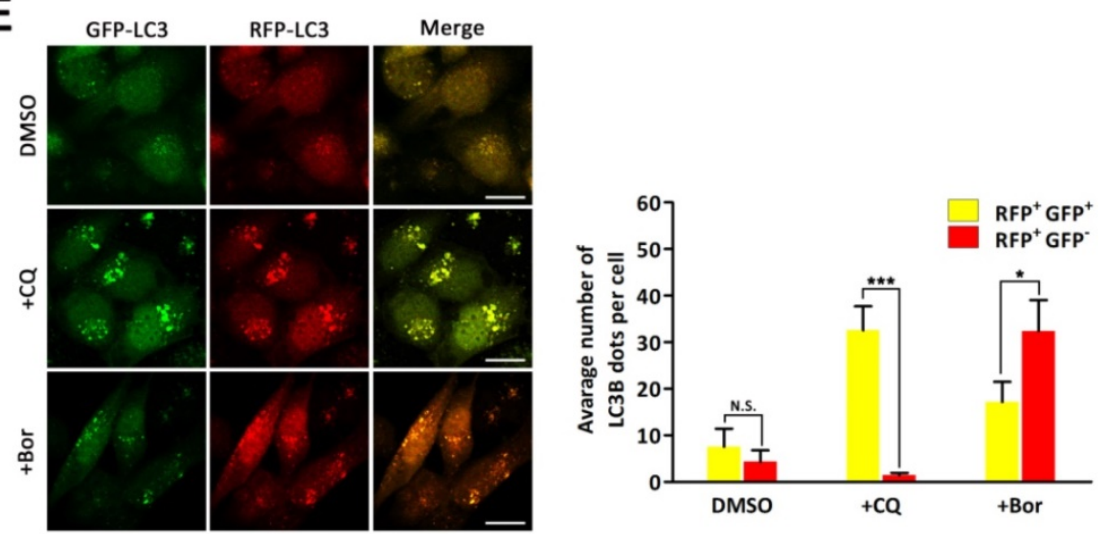

Figure 2. Bortezomib induces autophagic flux in PC cells. (A) MIAPaCa-2 and AsPC-1 cells were treated with the indicated concentrations of bortezomib for $24 \mathrm{~h}$. The indicated protein levels were analyzed by western blot. (B) MIAPaCa-2 and AsPC-1 cells transfected with GFP-LC3B were treated with bortezomib (100 nM) for 24 h. The number of GFP-LC3B dots in each cell was quantified, and at least 50 cells were included for each group. Scale bar: $20 \mu \mathrm{m}$. Each bar represents means \pm SD of three separate experiments. ${ }^{* *} \mathrm{p}<0.001$. (C) MIAPaCa-2 and AsPC-1 cells treated with DMSO $(<0.1 \%)$ or bortezomib $(100 \mathrm{nM})$ for $24 \mathrm{~h}$ and imaged by transmission electron microscope. Representative images of cells are shown. A magnified view of the electron photomicrograph shows a characteristic autophagosome. Arrowhead, autophagic vacuoles; N, nuclear; Scale bar: $1 \mu \mathrm{m}$. Quantification of the number of autophagic vacuoles from at least 20 randomly selected areas is shown. *** $<<0.001$. (D) MIAPaCa-2 and AsPC-1 cells were either untreated or treated with bortezomib $(100 \mathrm{nM})$ for $24 \mathrm{~h}$ in the presence or absence of $\mathrm{CQ}(10 \mu \mathrm{M})$. The indicated protein levels were analyzed by western blot. (E) MIAPaCa-2 cells transfected with GFP-mRFP-LC3B were treated with DMSO (<0.1\%), CQ $(10 \mu M)$, or bortezomib $(100 \mathrm{nM})$ for $24 \mathrm{~h}$, and then observed for the change of both green and red fluorescence using a confocal microscope. Scale bar: $20 \mu \mathrm{m}$. The numbers of acidified autophagosomes (GFP-/RFP+) versus neutral autophagosomes (GFP+/RFP+) per cell in each condition were quantified. Data are presented as mean \pm SD from three separate experiments. N.S, not significant; * ${ }^{*}<0.05 ; * * * p<0.001$.

the protein level and attenuated bortezomib-induced LC3B-II formation in both MIAPaCa-2 and AsPC-1 cells. Additionally, CHOP knockdown markedly reduced bortezomib-induced apoptosis compared with control siRNA (Fig. 3E). Similarly, the ER stress inhibitor TUDCA significantly decreased bortezomibinduced Bip and CHOP expression, attenuated LC3B-II formation and decreased bortezomib-induced apoptosis (Fig. 3F). Conversely, the addition of a typical ER stress inducer, TM, with bortezomib significantly increased LC3B-II formation and the apoptotic ratio compared with either agent alone (Fig. 3F).
Previous studies have reported that releasing calcium from the ER into the cytosol and mitochondria promotes apoptosis due to mitochondrial calcium overload [35]. To determine whether increased intracellular calcium is important for bortezomib-induced PC cell apoptosis, we first detected changes in subcellular calcium accumulation by Immunofluorescence. Interestingly, confocal microscopy indicated that bortezomib treatment induced a remarkable increase in cytosolic calcium colocalized within mitochondria in MIAPaCa-2 and AsPC-1 cells (Fig. 3G). Additionally, inhibiting calcium accumulation with BAPTA-AM (an 
A

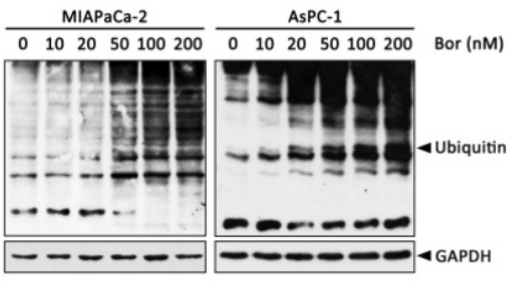

B

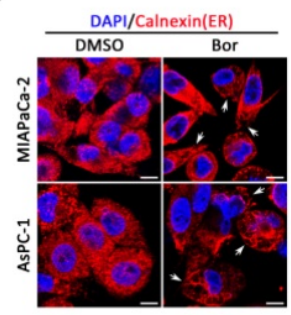

C

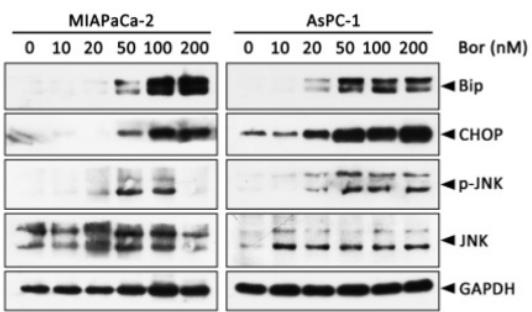

D

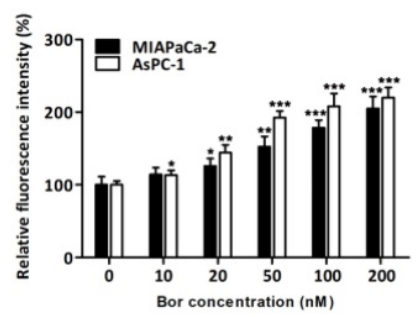

G

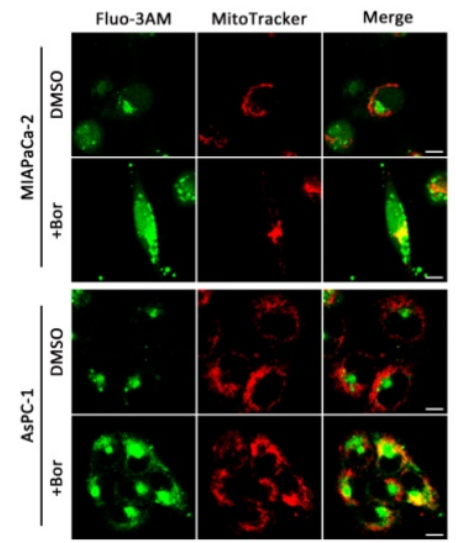

E
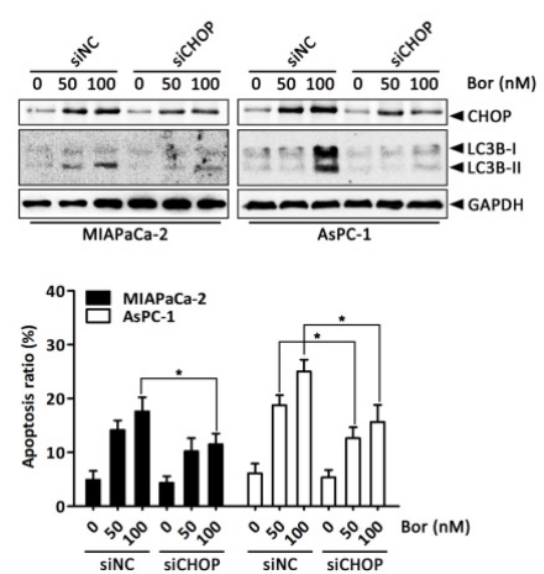

$\mathrm{H}$

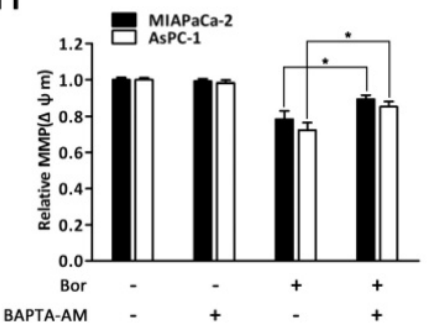

F
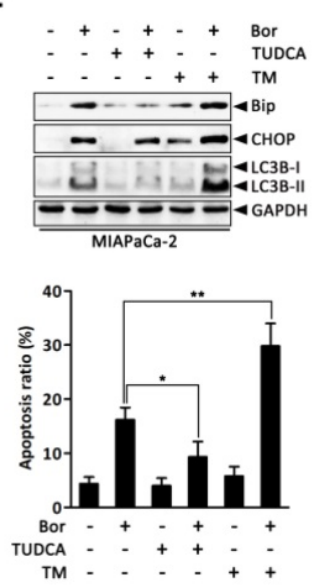

I

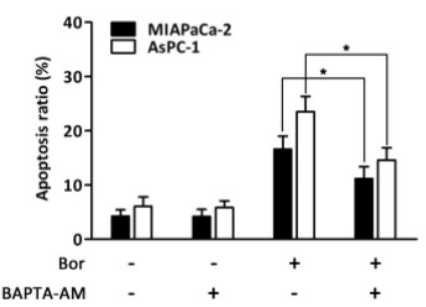

Figure 3. Bortezomib-induced apoptosis and autophagy are associated with ER stress. (A) Western blot analysis of ubiquitin protein levels after MIAPaCa-2 and AsPC-1 cells were treated with the indicated concentrations of bortezomib for $24 \mathrm{~h}$. (B) MIAPaCa-2 and AsPC-1 cells were treated with DMSO ( $<0.1 \%$ ) or bortezomib $(100$ $\mathrm{nM}$ ) for $24 \mathrm{~h}$ followed by immunostaining with an anti-calnexin antibody. Nuclei are counterstained with DAPI (blue). Arrows indicate dilated ER cavities. Scale bar: $10 \mu \mathrm{m}$. (C) Western blot analysis of ER stress-related protein levels after MIAPaCa-2 and AsPC-1 cells were treated as described in (A). (D) MIAPaCa-2 and AsPC-1 cells were treated as described in (A), and the cytosolic calcium levels was determined by Fluo-3/AM staining and analyzed by flow cytometry. Each bar represents means \pm SD of three separate experiments. ${ }^{*} \mathrm{p}<0.05 ;{ }^{*} \mathrm{p}<0.01 ;{ }^{* * *} \mathrm{p}<0.001$. (E) MIAPaCa-2 and AsPC-1 cells were transfected with CHOP siRNAs for $48 \mathrm{~h}$ and then cells were treated with the indicated concentrations of bortezomib for an additional $24 \mathrm{~h}$. The indicated protein levels (upper panel) and the apoptotic cells (lower panel) were determined by western blot and annexin V/PI double staining assay, respectively. Each bar represents means \pm SD of three separate experiments. *p $<0.05$. (F) MIAPaCa-2 cells were pretreated with TUDCA $(1 \mathrm{mM})$ or TM $(10 \mu \mathrm{g} / \mathrm{ml})$ for $30 \mathrm{~min}$, and then exposed to bortezomib $(100 \mathrm{nM})$ for $24 \mathrm{~h}$. The indicated protein levels (upper panel) and the apoptotic cells (lower panel) were determined by western blot and annexin V/PI double staining assay, respectively. Each bar represents means \pm SD of three separate experiments. * $<0.05$; $* * p<0.01$. (G) MIAPaCa-2 and AsPC-1 cells were treated with DMSO $(<0.1 \%)$ or bortezomib $(100 \mathrm{nM})$ for $24 \mathrm{~h}$. After incubated with MitoTracker Red for 30 min, cells were stained with Fluo-3/AM and observed under a confocal microscope. Scale bar: $10 \mu \mathrm{m}$. (H, I) MIAPaCa-2 and AsPC-1 cells were treated with bortezomib (100 $\mathrm{nM}$ ) for $24 \mathrm{~h}$ in the presence or absence of BAPTA-AM $(10 \mu \mathrm{M})$. The $\Delta \psi \mathrm{m}(\mathrm{H})$ and the apoptotic cells $(\mathrm{I})$ were detected by flow cytometry using JC-1 and annexin V/PI double staining assay, respectively. Each bar represents means \pm SD of three separate experiments. ${ }^{*} \mathrm{p}<0.05$.

intracellular calcium chelator) pretreatment significantly suppressed the bortezomib-induced loss of $\Delta \psi \mathrm{m}$ and activation of apoptosis (Fig. $3 \mathbf{H}$ and $\mathbf{~}$ ). Taken together, these results indicate that bortezomib triggered ER stress, which subsequently induced apoptosis and autophagy in PC cells.

\section{Autophagy protects against bortezomib-induced apoptosis in PC cells.}

Having clearly established that bortezomib activates autophagy in PC cells, we next sought to determine how autophagy contributes to cell death.
To explore the biological role of autophagy in bortezomib-induced cell death, CQ was used to block lysosomal function and prevent complete autophagy. As shown in Fig. 4A, both MIAPaCa-2 and AsPC-1 cells had more significantly inhibited growth after combined bortezomib and CQ treatment compared with bortezomib alone. Furthermore, CQ markedly increased bortezomib-induced caspase- 3 and PARP cleavage (Fig. 4B). To exclude non-specific CQ effects, we silenced Atg5, an essential autophagy gene, via siRNA and analyzed the relationship between apoptosis and autophagy. Downregulating Atg5, 
A

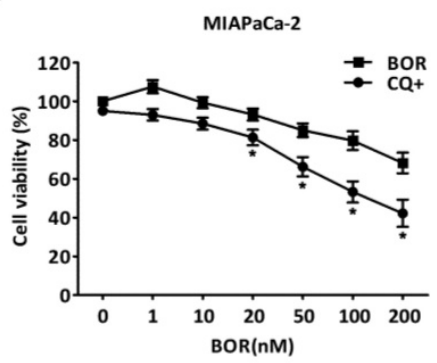

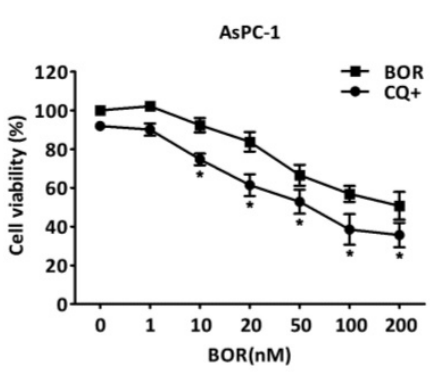

B

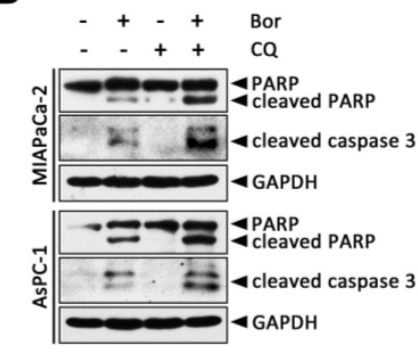

C

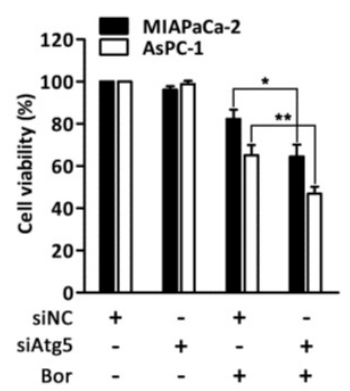

D

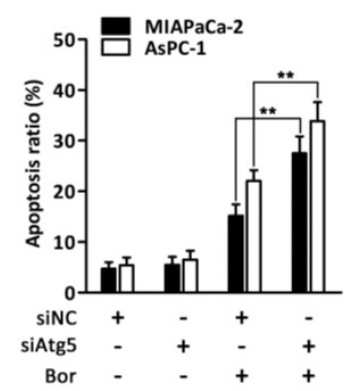

E

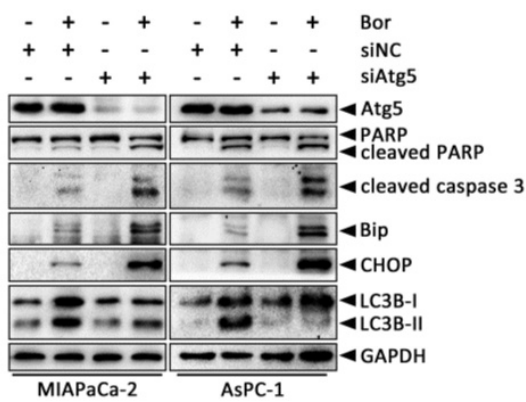

Figure 4. Autophagy protects against bortezomib-induced apoptosis in PC cells. (A) MIAPaCa-2 and AsPC-1 cells were treated with the indicated concentrations of bortezomib for $24 \mathrm{~h}$ in the presence or absence of CQ $(10 \mu \mathrm{M})$. The cell viability was determined by CCK-8 assay. Each data point represents means \pm SD of three separate experiments. ${ }^{*} \mathrm{p}<0.05$. (B) MIAPaCa-2 and AsPC-1 cells were either untreated or treated with bortezomib $(100 \mathrm{nM})$ for $24 \mathrm{~h}$ in the presence or absence of CQ (10 $\left.\mu \mathrm{M}\right)$. The indicated protein levels were analyzed by western blot. (C, D) MIAPaCa-2 and AsPC-1 cells were transfected with Atg5 siRNAs for $48 \mathrm{~h}$ and then cells were treated with bortezomib $(100 \mathrm{nM})$ for an additional $24 \mathrm{~h}$. The cell viability $(\mathrm{C})$ and the apoptotic cells $(\mathrm{D})$ were determined by CCK-8 and annexin V/PI double staining assay, respectively. Each bar represents means \pm SD of three separate experiments. ${ }^{*} \mathrm{p}<0.05$; ${ }^{* *} \mathrm{p}<0.01$. (E) MIAPaCa- 2 and AsPC-1 cells were treated as described in (C), and the indicated protein levels were analyzed by western blot.

which significantly decreased LC3B-II accumulation in bortezomib-treated cells, enhanced the sensitivity to bortezomib-induced cell death and increased cleaved caspase-3 and PARP levels (Fig. 4C-E). Interestingly, inhibiting autophagy by silencing Atg5 also resulted in a significant increase in bortezomib-induced Bip and CHOP expression in MIAPaCa-2 and AsPC-1 cells (Fig. 4E).

Beclin1 is a key initiator of autophagy in mammalian cells [14]; thus, the finding that bortezomib treatment did not stimulate increased Beclin1 expression prompted us to investigate the role of Beclin1 in bortezomib-induced autophagy. Surprisingly, silencing Beclin1 via Beclin1 siRNA did not affect the accumulation of LCB3-II in bortezomib-treated cells, nor show any significant effect on bortezomib-induced apoptosis (Fig. S4). Collectively, these data indicated that bortezomib induced protective autophagy in PC cells via a Beclin1-independent pathway, which alleviates ER stress and reduces apoptosis.

\section{ROS elevation is a critical event in bortezomib-induced ER stress and autophagy in PC cells.}

ER stress, autophagy and oxidative stress are closely linked events. Bortezomib reportedly induces ROS generation in cancer cells [36, 37]. Therefore, to gain further insight into the mechanism of bortezomib-induced ER stress and autophagy in PC cells, we assessed intracellular ROS levels using the fluorescent probe DCFH-DA. There was a significant increase in intracellular ROS levels in MIAPaCa-2 and AsPC-1 cells following bortezomib treatment (Fig. 5A). This increase in bortezomib-induced ROS could be blocked by pretreating cells with the ROS scavenger, NAC (Fig. 5B). Moreover, NAC also significantly attenuated bortezomib-induced apoptosis and decreased cleaved caspase- 3 and PARP levels (Fig. 5C and D). We then focused our attention onto whether ROS generation was associated with bortezomib-induced ER stress and autophagy. As expected, pretreating cells with NAC not only attenuated bortezomib-induced increased intracellular calcium levels (Fig. S5), but also decreased bortezomib-induced Bip and CHOP expression (Fig. 5D). Furthermore, NAC significantly decreased LC3B-II accumulation (Fig. 5D). These results indicated that ROS generation plays a critical role in bortezomib-induced ER stress and autophagy in PC cells.

\section{Autophagy and Nrf2 signaling are adaptive mechanisms for bortezomib-induced ROS production.}

It has been proposed that augmented autophagic removal of oxidatively damaged organelles and proteins is a defense against oxidative stress [38]. To 
A

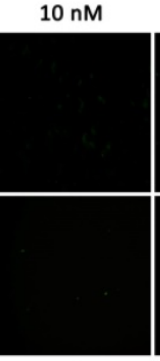

$20 \mathrm{nM}$
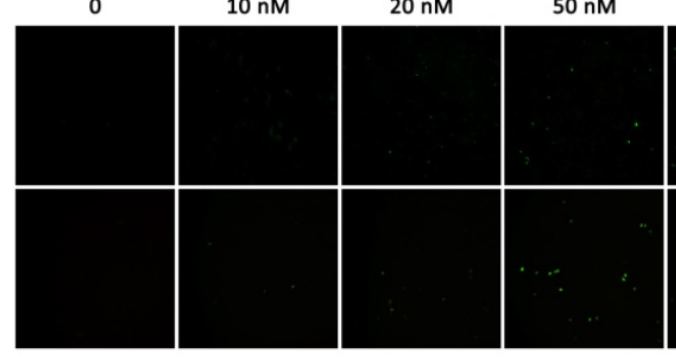

$100 \mathrm{nM}$
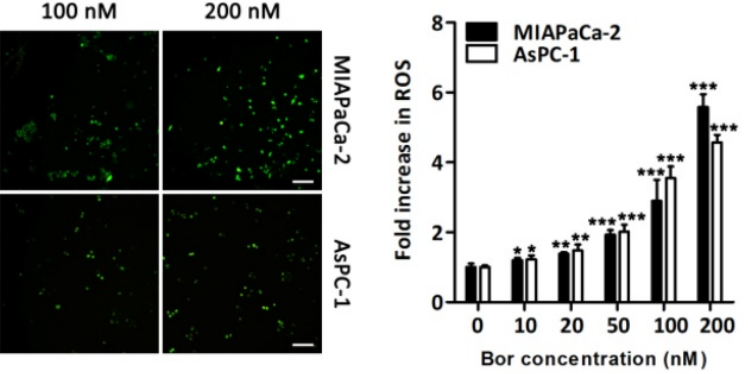

B

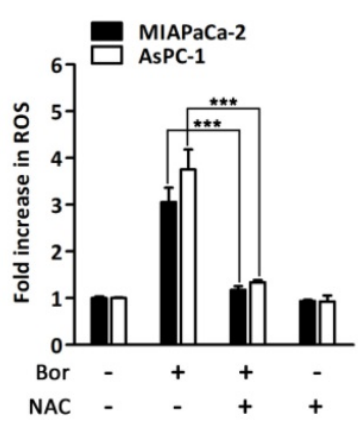

C

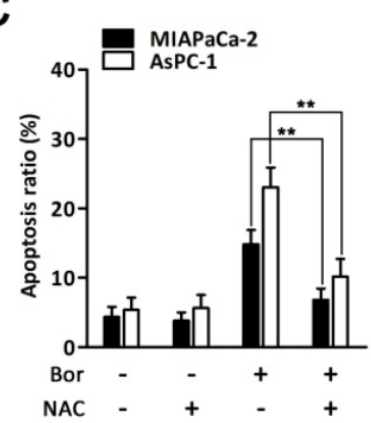

D

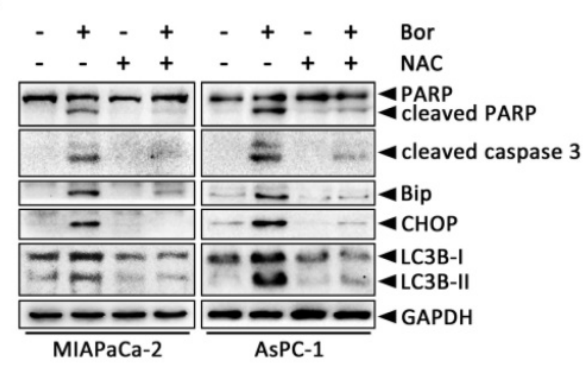

Figure 5. ROS elevation is a critical event in bortezomib-induced ER stress and autophagy in PC cells. (A) MIAPaCa-2 and AsPC-1 cells were treated with the indicated concentrations of bortezomib for $24 \mathrm{~h}$. After incubation with DCFH-DA, cells were washed and examined by fluorescence microscope. Representative images of cells from three independent experiments are shown. Scale bar: $100 \mu \mathrm{m}$. Fluorescence intensity was measured by flow cytometry. Fold increase in ROS production in treated cells was compared with untreated cells. Each bar represents means \pm SD of three separate experiments. *p $<0.05$; **p $<0.01$; ***p $<0.001$. (B) MIAPaCa-2 and AsPC-1 cells were pretreated with NAC $(5 \mathrm{mM})$ for $30 \mathrm{~min}$, and then exposed to bortezomib $(100 \mathrm{nM})$ for $24 \mathrm{~h}$. Fold increase in ROS was determined by DCFH-DA staining and analyzed by flow cytometry. Each bar represents means \pm SD of three separate experiments. ***p $<0.001$. (C) Apoptotic cells were detected by flow cytometry using annexin V/PI double staining after cells were treated as described in (B). Each bar represents means \pm SD of three separate experiments. **p $<0.01$. (D) MIAPaCa-2 and AsPC-1 cells were treated as described in (B), and the indicated protein levels were analyzed by western blot.

determine whether autophagy could modulate bortezomib-induced ROS production in PC cells, we quantified intracellular ROS levels after combined treatment with bortezomib and CQ. As shown in Fig. 6A, ROS levels were markedly increased in MIAPaCa-2 and AsPC-1 cells treated with the combination of bortezomib and CQ compared with bortezomib alone. Similarly, inhibiting autophagy by silencing Atg5 enhanced bortezomib-induced ROS levels (Fig. 6B).

The transcription factor Nrf2 is the main adaptive cellular regulator that protects against oxidative stress $[23,24]$. We next investigated whether Nrf2 was involved in bortezomib-induced ROS production in PC cells. As shown in Fig. 6C, levels of $\mathrm{Nrf} 2$ and its downstream targets HO-1 and NQO1 increased following bortezomib treatment in MIAPaCa-2 and AsPC-1 cells. Moreover, levels of Nrf2 in the nuclear fraction were also dramatically stimulated after bortezomib treatment, indicating the nuclear translocation of Nrf2, where it stimulated the transcription of its target genes. Additionally, Nrf2 knockdown via siRNA resulted in a slightly increased basal ROS levels, which were further elevated compared with scrambled siRNA-transfected cells following bortezomib treatment, indicating that the Nrf2 pathway participated in bortezomib-induced
ROS generation (Fig. 6D). Intriguingly, in cells where Nrf2 was downregulated, both basal and bortezomibinduced LC3B-II formation were also elevated (Fig. $6 \mathrm{E})$, indicating the compensatory role of autophagy under increased ROS conditions. Consistent with these results, we found a significant increase in GFP-LC3B puncta in the cytoplasm of cells transfected with Nrf2 siRNA compared with scrambled siRNA upon bortezomib exposure (Fig. S6). Together, these findings indicate that both autophagy and Nrf2 signaling are adaptive mechanisms that are activated upon bortezomib-induced ROS generation in PC cells.

\section{Combined inhibition of autophagy and Nrf2 pathway augments bortezomib-induced apoptosis in PC cells.}

Given that our previous experiments showed that bortezomib increased intracellular ROS levels, which then induced autophagy and Nrf2 pathway activation, we next queried whether the dual activation of autophagy and the Nrf2 antioxidant system caused a potent cooperative effect to counteract bortezomib in PC cells. As shown in Fig. 7A and B, compared with individually knocking down Atg5 or Nrf2, combined Atg5 and Nrf2 knockdown further augmented bortezomib-induced ROS levels and apoptosis rates in MIAPaCa- 2 and 
AsPC-1 cells. More remarkably, double Atg 5 and Nrf2 knockdown cooperatively enhanced bortezomibinduced Bip and CHOP expression as well as caspase-9, caspase-3 and PARP cleavage compared with single Atg5 or Nrf2 knockdown (Fig. 7C and Fig. S7), indicating that simultaneously suppressing autophagy and the Nrf2 pathway enhances Bortezomib-induced ER stress and apoptosis at least in an additive manner in PC cells. Of note, Atg5-specific siRNA caused a marked increase in bortezomib-induced Nrf2 expression compared with scrambled siRNA-transfected cells (Fig. 7C and Fig. S7), suggesting that suppressing autophagy increases the cell's reliance on the Nrf2 pathway during bortezomib treatment. Conversely, pretreating cells with NAC or TUDCA strikingly attenuated the cell death induced by combination treatment (Fig. 7D), indicating that both ER stress and ROS play a key role in the cytotoxicity of the combination treatment. From these results, we conclude that combined inhibition of autophagy and Nrf2 pathway rendered PC cells vulnerable to bortezomib by increasing ROS production and ER stress.

\section{Discussion}

Herein, we report that treating PC cells with bortezomib triggers ER stress and subsequently induce autophagy and apoptosis. Bortezomib also increased intracellular ROS, which plays a critical role in bortezomib-induced ER stress and apoptosis. Moreover, autophagy activation functions as a compensatory mechanism to eliminate bortezomibinduced ROS and resist ER stress-mediated apoptosis. Additionally, Nrf2-mediated antioxidative responses, which negatively impact bortezomib-induced autophagy, also protect against bortezomib-induced ROS production. Finally, we found that the combined inhibition of autophagy and Nrf2 signaling resulted in an additive enhancement of bortezomib-induced apoptosis by promoting ROS accumulation and ER stress in PC cells.

Proteasome inhibition appears to prevent the clearance of misfolded proteins through the ERAD system, which leads to protein aggregation in the ER, triggering ER stress-mediated apoptosis. Indeed, it has been widely reported that ER stress is involved in the antitumor effects of proteasome inhibitors [6, 8]. Consistent with previous studies, our results
A

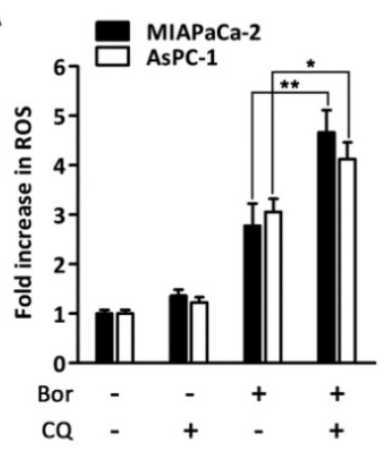

B

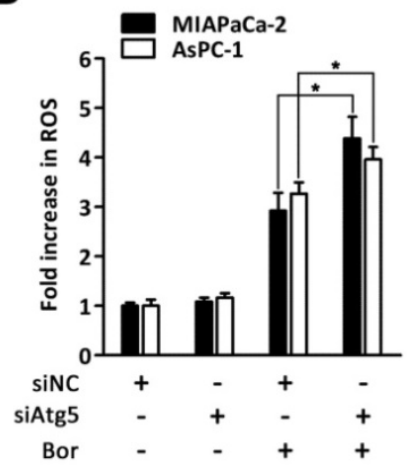

C

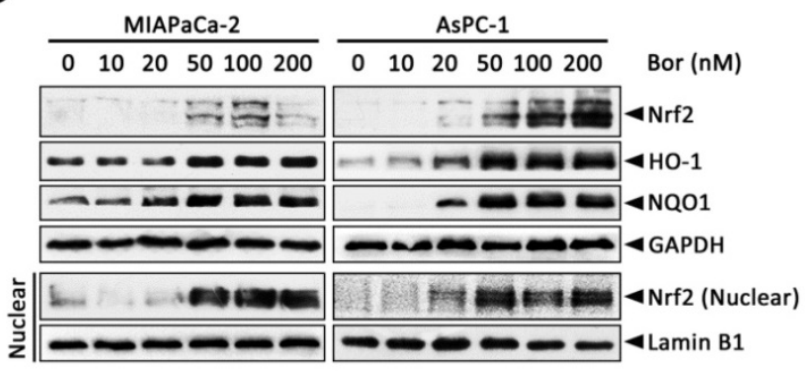

D

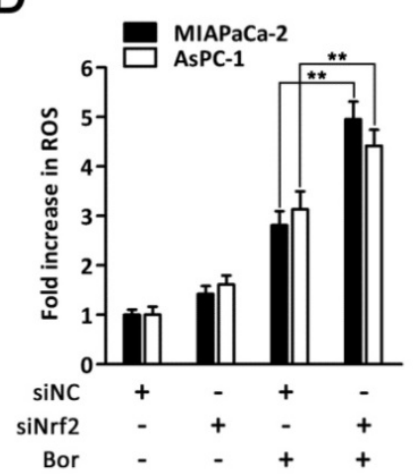

E

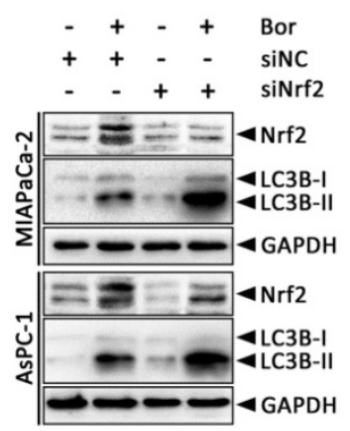

Figure 6. Autophagy and Nrf2 signaling are adaptive mechanisms for bortezomib-induced ROS production. (A) MIAPaCa- 2 and AsPC-1 cells were treated with bortezomib $(100 \mathrm{nM})$ for $24 \mathrm{~h}$ in the presence or absence of CQ $(10 \mu \mathrm{M})$. Fold increase in ROS was determined by DCFH-DA staining and analyzed by flow cytometry. Each bar represents means \pm SD of three separate experiments. ${ }^{*} \mathrm{p}<0.05$; ${ }^{* *} \mathrm{p}<0.01$. (B) MIAPaCa-2 and AsPC-1 cells were transfected with Atg5 siRNAs for $48 \mathrm{~h}$ and then cells were treated with bortezomib $(100 \mathrm{nM})$ for an additional $24 \mathrm{~h}$. Fold increase in ROS was determined by DCFH-DA staining and analyzed by flow cytometry. Each bar represents means \pm SD of three separate experiments. **p $<0.01$. (C) Western blot analysis of Nrf2, HO-1, NQO1 and Nrf2 in nuclear fractions levels after MIAPaCa-2 and AsPC-1 cells were treated with the indicated concentrations of bortezomib for $24 \mathrm{~h}$. Lamin B1 was used as nuclear internal control. (D) MIAPaCa-2 and AsPC-1 cells were transfected with Nrf2 siRNAs for $48 \mathrm{~h}$ and then cells were treated with bortezomib $(100 \mathrm{nM})$ for an additional $24 \mathrm{~h}$. Fold increase in ROS was determined by DCFH-DA staining and analyzed by flow cytometry. Each bar represents means \pm SD of three separate experiments. **p $<0.01$. (E) MIAPaCa-2 and AsPC-1 cells were treated as described in (D), and the indicated protein levels were analyzed by western blot. 
A

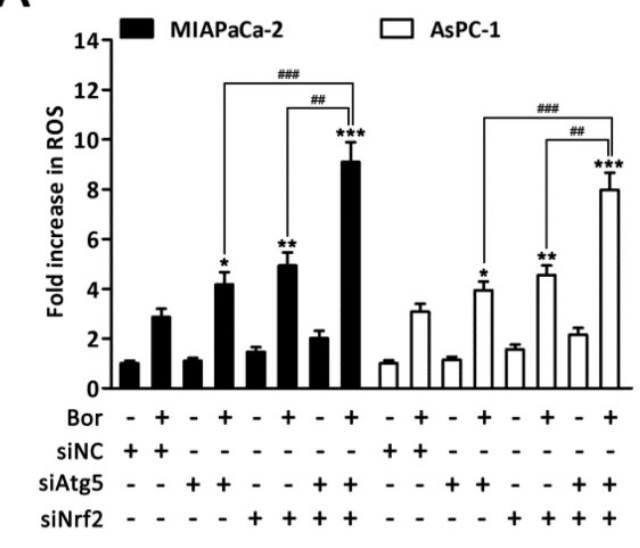

C

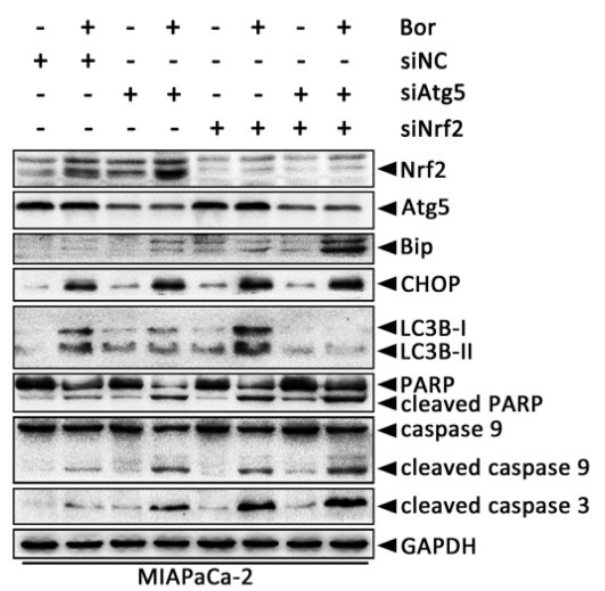

B

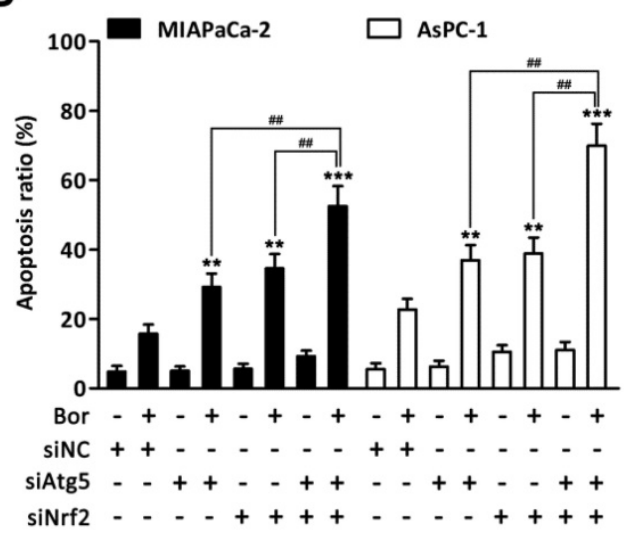

D

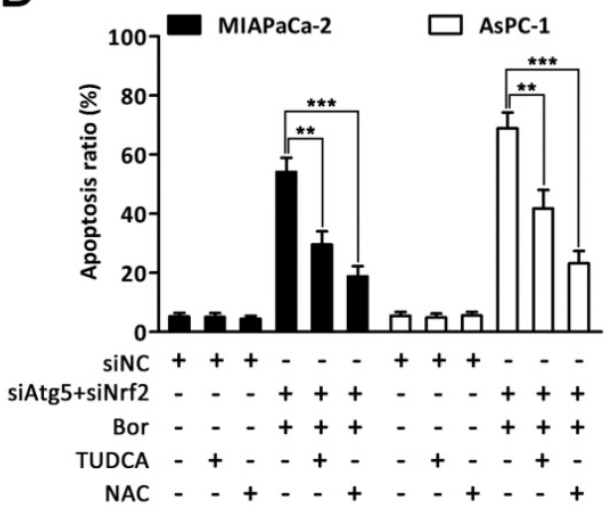

Figure 7. Combined inhibition of autophagy and Nrf2 pathway augments bortezomib-induced apoptosis in PC cells. (A, B) MIAPaCa-2 and AsPC-1 cells were transfected with Atg5 or Nrf2 siRNA, or combinations thereof for $48 \mathrm{~h}$, and then cells were treated with bortezomib (100 nM) for an additional $24 \mathrm{~h}$. Fold increase in ROS (A) and the apoptotic cells (B) were determined by DCFH-DA and annexin V/PI double staining assay, respectively. Each bar represents means \pm SD of three separate experiments. $*_{p}<0.05$; **p $<0.01$; *** $p<0.001$; $\mathrm{N}_{\mathrm{p}}<0.01$; $\mathrm{m}<0.001$. (C) MIAPaCa-2 cells were treated as described in (A), and the indicated protein levels were analyzed by western blot. (D) MIAPaCa-2 and AsPC-1 cells were transfected with scrambled siRNA or Atg5 plus Nrf2 siRNA, and then untreated or treated with bortezomib (100 nM) for $24 \mathrm{~h}$ in the absence or presence of TUDCA $(1 \mathrm{mM})$ or NAC $(5 \mathrm{mM})$. The apoptotic cells were determined by annexin V/PI double staining assay. Each bar represents means \pm SD of three separate experiments. **p $<0.01 ; * * * \mathrm{p}<0.001$.

indicated that bortezomib treatment increased the levels of both ubiquitinated proteins and ER stress-associated proteins in PC cells. Moreover, bortezomib increased JNK activation and intracellular calcium levels, both of which are indicative of ER stress [11]. These findings suggest that bortezomib induces ER stress in PC cells. CHOP is a crucial mediator of apoptosis following ER stress [11]. We found that downregulating CHOP via siRNA blocked bortezomib-induced apoptosis. Furthermore, pretreatment with the ER stress inhibitor TUDCA dramatically attenuated bortezomib-induced apoptosis; whereas the addition of a typical ER stress inducer TM promoted bortezomib-induced apoptosis, leading to the suggestion that ER stress is directly involved in bortezomib-induced apoptosis.

Notably, bortezomib appears to induce apoptosis in PC cells through a mitochondrial apoptotic pathway, evidenced by the loss of $\Delta \psi \mathrm{m}$, release of cytochrome $c$, as well as activation of caspase-9, caspase-3 and PARP. Bcl-2 family proteins regulate the mitochondrial apoptosis pathway by interacting with mitochondrial membranes to control the $\Delta \psi \mathrm{m}[39,40]$. Our data suggested that bortezomib dramatically decreased levels of the Bcl-2 family proteins Bcl-2, XIAP, and Bcl-XL. Similar to these results, bortezomib-mediated mitochondrial apoptosis was also observed in lung cancer cells [36]. Increasing intracellular calcium levels has been reported to play an important role in activating mitochondrial apoptosis due to mitochondrial calcium overload [35]. The accumulation of calcium into the mitochondria strictly depends on the ER, which serves as the primary organelle for calcium homeostasis [35]. In this study, confocal microscopy confirmed that bortezomib markedly increased mitochondrial calcium accumulation in PC cells, suggesting bortezomib-induced ER stress causes calcium to leak from the ER lumen into the mitochondria. Furthermore, BAPTA-AM pretreatment significantly inhibited bortezomib-induced $\Delta \psi \mathrm{m}$ loss and apoptosis, indicating that bortezomib- 
induced PC cell apoptosis may be mediated through increased levels of intracellular calcium. However, the $\Delta \psi \mathrm{m}$ stabilizer CsA only partially blocked bortezomib-induced apoptosis, suggesting that additional mechanisms are involved in this process.

Previous studies have reported that proteasome inhibitors induce autophagy in several cancer models $[17,29]$. In accordance with these data, we found that bortezomib treatment of PC cells induced the accumulation of LC3B-II and autophagic vacuoles, promoted GFP-LC3B redistribution, and stimulated autophagic flux, all of which confirmed that autophagy was activated. Moreover, bortezomibinduced autophagy played a protective role in PC cells, as evidenced by the increased rate of bortezomib-induced apoptosis in autophagyinhibited cells. Beclin-1 is part of the class III phosphatidylinositol 3-kinase (PI3K) complex that is involved in autophagosome formation [14]. One particularly interesting observation is the discovery that Beclin1 knockdown had no obvious effects on bortezomib-induced apoptosis or autophagy, indicating that bortezomib induced autophagy in a Beclin1-independent manner. Similar to our findings, proteasome inhibitors have also been reported to cause Beclin1-independent autophagy in thyroid cancer cells [41]. Accumulating data indicate that ER stress is a potent trigger of autophagy [13,32]. In these situations, autophagy counterbalances ER expansion, removes aggregated proteins, and thus, is cytoprotective. Indeed, pretreatment with the ER stress inhibitor TUDCA or transfecting CHOP-specific siRNA partially reduced bortezomib-induced LC3B-II accumulation, whereas the ER stress inducer TM promoted it. Additionally, inhibiting autophagy by Atg5 siRNA also increased bortezomib-induced ER stress-associated protein expression. Collectively, these findings strongly support the conclusion that ER stress precedes bortezomib-induced autophagy, which in turn ameliorates the ER stress.

Multiple lines of evidence have shown that, compared with their normal counterparts, cancer cells (including PC cells) seem to function with higher levels of intracellular ROS in culture and in vivo [18]. In fact, low ROS levels could promote the activities of several signaling proteins involved in cell proliferation and tumor progression through multiple mechanisms. However, if the ROS levels exceed a certain threshold, they may become cytotoxic [18, 19]. Previous studies have indicated that bortezomib may induce ROS generation as part of its mechanism of action [36, 37]. Likewise, our results showed that bortezomib increased ROS in PC cells, which may be a key mediator of bortezomib-induced apoptosis, as apoptosis was blocked by the ROS scavenger NAC.
Additionally, NAC pretreatment also attenuated the bortezomib-induced increase of intracellular calcium and the increased expression of Bip, CHOP and LC3B-II, suggesting ROS may play an important role in bortezomib-induced ER stress and autophagy in PC cells.

However, under persistent intrinsic oxidative stress, cancer cells become highly adapted to such ROS stress and develop a set of endogenous antioxidant capacities. These adaptive mechanisms not only activate ROS-scavenging systems to cope with the stress, but also make the malignant cells resistant to anticancer agents $[18,19]$. Several studies have suggested that resistance anticancer agents that induce intracellular ROS production is correlated with increased antioxidant capacity [18, 19]. Recently, increasing evidence has supported the function of autophagy under metabolic stress as a way to remove damaged organelles and accumulated proteins, which lowers intracellular ROS levels [38]. Consistent with previous observations, we found that suppressing autophagy with CQ of Atg5 knockdown increased bortezomib-induced ROS levels, indicating that autophagy maybe involved in protecting PC cells against bortezomib-induced oxidative stress. Additionally, the Nrf2-mediated antioxidant response is critical for maintaining cellular redox homeostasis [23, 24]. In our experiments, levels of Nrf2 and its downstream targets were dramatically increased under bortezomib treatment, and silencing Nrf2 resulted in a further elevation of bortezomib-induced ROS as well as increased LC3B-II expression. These results indicated that when faced with oxidative stress, and the Nrf2 pathway is suppressed, autophagy is further promoted to deal with excessive ROS. Interestingly, Atg5 knockdown also led to increased bortezomib-induced Nrf2 expression. Thus, we hypothesized that there could be a negative interaction between autophagy and the Nrf2 pathway when cells were stimulated with high-level ROS, as both are required to coordinately deal with ROS-mediated damage and promote PC cell survival. Indeed, our next experiments proved that simultaneously suppressing autophagy and Nrf2 signaling cooperatively augmented bortezomibinduced ROS, which subsequently enhanced bortezomib-induced apoptosis, as NAC pretreatment significantly attenuated the apoptosis rates induced by the combination treatments. Similar to our findings, it was previously reported that ionizing radiation-induced ROS promoted autophagy and Nrf2, which conferred radio-resistance in lung cancer cell [42].

In summary, this study suggests that autophagy and the Nrf2 antioxidant system, which both can 
lower intracellular ROS, are involved in the mechanism of bortezomib-resistance in PC cells. Combined with their negative interaction, we provide the basis for future preclinical and clinical trials exploring proteasome inhibitors and novel drug targets to co-inhibit autophagy and Nrf2 signaling as a combinatory therapeutic approach for PC.

\section{Materials and Methods}

\section{Cell culture and reagents}

Human pancreatic cancer cell lines MIAPaCa-2 and AsPC-1 were purchased from American Type Culture Collection (Manassas, VA, USA). All cells were maintained in Dulbecco's modified Eagle's medium (DMEM) or Roswell Park Memorial Institute 1640 (RPMI-1640) medium supplemented with 10\% fetal bovine serum (FBS), $2 \mathrm{mmol} / 1$ of glutamine, 100 units $/ \mathrm{ml}$ of penicillin and $100 \mathrm{mg} / \mathrm{ml}$ of streptomycin in a $5 \% \mathrm{CO}_{2}$ atmosphere at $37^{\circ} \mathrm{C}$. Bortezomib and cyclosporin A (CsA) were purchased from Santa Cruz Biotechnology (Santa Cruz, CA, USA). Chloroquine (CQ), BAPTA-AM and N-acetyl-L-cysteine (NAC) were purchased from Sigma-Aldrich (St. Louis, MO, USA). Tauroursodeoxycholic acid (TUDCA) and tunicamycin (TM) were obtained from Calbiochem (Merck, Darmstadt, Germany). JC-1, Fluo-3/AM and 2', 7'-dichlorofluorescin diacetate (DCFH-DA) were obtained from Beyotime (Haimen, China). MitoTracker Red was obtained from Invitrogen (Carlsbad, CA, USA). All commercial antibodies were purchased from the following resources: anti-LC3B, anti-Beclin1, anti-Atg5, anti-Atg7, anti-Calnexin, anti-ubiquitin, anti-CHOP, anti-Bip, anti-p-JNK, anti-PARP, anti-cleaved caspase-3, anti-caspase-9 and anti-Lamin B1 antibodies were from Cell Signaling Technology (Beverly, MA, USA); anti-JNK antibody was from Santa Cruz Biotechnology (Santa Cruz, CA, USA); anti-Nrf2, anti-HO-1, anti-NQO1, anti-Bcl-XL, anti-Bcl-2 and anti-cytochrome $\mathrm{c}$ antibodies were from Epitomics (Burlingame, CA, USA); anti-GAPDH antibody was from Proteintech Group (Chicago, IL, USA); anti-XIAP antibody and horseradish peroxidase-conjugated secondary antibodies (goat anti-rabbit and goat anti-mouse) were purchased from Beyotime (Haimen, China). The chemicals were dissolved in either appropriate media solution or dimethyl sulfoxide (DMSO) and then treated at the required working dilution. All chemicals were handled in accordance with the supplier's recommendations.

\section{Cell viability assay}

The cell viability was measured using CCK-8 assay (Dojindo Laboratories, Kumamoto, Japan) according to the manufacturer's instructions. Briefly,
Cells (5,000 per well) were plated in 96-well plate. After $24 \mathrm{~h}$, cells were treated with the chemical agents as indicated in the figure legends. DMSO was used as vehicle. After $10 \mu \mathrm{L}$ WST-8 dye was add to each well, cells were incubated at $37^{\circ} \mathrm{C}$ with $5 \% \mathrm{CO}_{2}$ for $2 \mathrm{~h}$ and the absorbance was finally measured at $450 \mathrm{~nm}$ with a microplate reader (Bio-tek Instruments, VT, USA).

\section{Colony formation assay}

To determine the long-term effects of drug treatment on cell colony formation, cells (500 cells) were seeded in a 6-well plate and treated as indicated in the figure legends. After rinsing with fresh medium, cells were incubated for 14 days and photographed after staining with $1 \%(\mathrm{w} / \mathrm{v})$ crystal violet in phosphate-buffered saline (PBS) including $25 \%$ methanol. The number of colonies, defined as $\geq$ 50 cells/colony, was counted and compared with untreated cells.

\section{Annexin V-FITC/propidium iodide (PI) apoptosis assay}

Cell apoptosis was detected following specific treatments by staining using the Annexin V-FITC/PI apoptosis kit (Becton Dickinson, San Jose, CA, USA) according to the manufacturer's instructions. Briefly, cells were harvested after treatment and resuspended in binding buffer. Aliquots of $10^{5}$ cells were mixed with $5 \mu \mathrm{L}$ each of annexin V-FITC and PI solution for $15 \mathrm{~min}$ at room temperature in the dark. After incubation, $400 \mu \mathrm{L}$ binding buffer was added, and cells were analyzed by FACS-Calibur flow cytometer (Becton Dickinson). Cell population in the lower left quadrant (Annexin V-/PI-) represents the live cells, population in the lower right quadrant (Annexin $\left.\mathrm{V}^{+} / \mathrm{PI}-\right)$ represents the early apoptotic cells, and population in the upper right quadrant (Annexin $\mathrm{V}^{+} / \mathrm{PI}^{+}$) represents the late apoptotic or dead cells.

\section{Mitochondrial transmembrane potential assessment}

Mitochondrial membrane potential (MMP, $\Delta \psi \mathrm{m}$ ) was analyzed by a fluorescent dye JC-1. Briefly, after indicated treatments, cells were incubated with JC-1 staining solution $(5 \mu \mathrm{g} / \mathrm{mL})$ for $20 \mathrm{~min}$ at $37^{\circ} \mathrm{C}$. Cells were then rinsed twice with PBS and the fluorescence of separated cells was detected by FACS-Calibur flow cytometer (Becton Dickinson, San Jose, CA, USA).

\section{Protein isolation and western blot analysis}

After treatment with different conditions as described in the figure legends, cells were harvested and lysed on ice in RIPA buffer (Beyotime, Haimen, China). After centrifuged at $12000 \mathrm{~g}$ for $15 \mathrm{~min}$, the clear supernatant was collected and used as the cell protein extract. For preparation of cytosolic and 
mitochondrial fractions, cells were collected and lysed in lysis buffer using mitochondria/cytosol fractionation kit (Beyotime, Haimen, China). Cytosol and mitochondrial extracts were isolated according to the manufacturer's protocol. Nuclear protein was extracted according to the manufacturer's instructions of the nuclear/cytoplasmic protein extraction kit (Beyotime, Haimen, China). Protein concentration was determined using the bicinchoninic acid protein assay kit (Beyotime, Haimen, China). Equivalent amounts of protein $(30 \mu \mathrm{g})$ from each sample were subjected to electrophoresis on a SDS-polyacrylamide gel, and then transferred on to PVDF membranes (Millipore, Bedford, MA, USA). Target antigens were detected with primary antibodies and subsequently secondary antibodies. Immunoreactive bands were then developed using an ECL chemiluminescence reagent (Pierce, Rockford, IL, USA), according to the manufacturer's instruction. The density of each band was measured using the public domain NIH Image software (open source Image J software available at http://rsb.info.nih.gov/ij/).

\section{Immunofluorescence and confocal laser-scanning microscopy}

After transfection with GFP-LC3B or GFP-mRFP-LC3B, cells were grown on glass coverslips. Following designated treatments, the fluorescent autophagy marker GFP-LC3B or GFP-mRFP-LC3B were observed under a confocal microscope (OLYMPUS, FluoView FV1000). The average number of GFP-LC3B dots per cell was determined from 3 independent experiments. 10 random fields representing 200 cells were counted on each coverslide.

For immunofluorescence, cells were seeded on sterile cover glasses placed in the 6-well plates. After designated treatments, cells were fixed with $4 \%$ formaldehyde for $15 \mathrm{~min}$ followed by permeabilization with $0.1 \%(\mathrm{w} / \mathrm{v})$ Triton X-100 for 10 min. Fixed cells were washed and blocked with $2 \%$ BSA for $30 \mathrm{~min}$, then incubated with primary antibodies against Calnexin $4^{\circ} \mathrm{C}$ overnight. After washing twice with PBS, antibody was visualized with Cy3-conjugated secondary antibody. Subsequently, cells were counterstained with 6-diamino-2-phenylindole (DAPI, Sigma) and observed under a confocal microscope (OLYMPUS, FluoView FV1000).

To detect the localization of intracellular calcium and mitochondrion, cells were grown on glass coverslips. Following designated treatments, the cells were first stained with MitoTracker Red $(50 \mathrm{nM})$ at $37^{\circ} \mathrm{C}$ for $30 \mathrm{~min}$. After washing with Hanks' balanced salt solution (HBSS), the cells were loaded with
Fluo-3/AM $(3 \mu \mathrm{M})$ staining solution at $37^{\circ} \mathrm{C}$ for $1 \mathrm{~h}$. Subsequently, the stained cells were immediately fixed with $4 \%$ paraformaldehyde for $10 \mathrm{~min}$ and observed under a confocal microscope (OLYMPUS, FluoView FV1000).

\section{Transmission electron microscopy}

After designated treatments, cells were first fixed in $3.0 \%$ glutaraldehyde, post-fixed with $1 \%$ osmium tetroxide for $1 \mathrm{~h}$ at $4^{\circ} \mathrm{C}$, dehydrated by a graded series of ethanol (30\%-100\%), and then polymerized by epoxy resin. Ultra-thin sections were cut and stained with $4 \%$ uranyl acetate and lead citrate. Finally, ultra-structure of the cells was observed under a Tecnai G2 F20 S-TWIN transmission electron microscope (FEI).

\section{Measurement of intracellular calcium}

The intracellular calcium level was measured by using the fluorescent dye Fluo-3/AM. Briefly, after indicated treatments, the cells were collected and incubated with Fluo-3/ AM $(3 \mu \mathrm{M})$ staining solution at $37^{\circ} \mathrm{C}$ for $1 \mathrm{~h}$. Then, the residual fluorescent dyes were washed with Hanks' balanced salt solution (HBSS) and the fluorescence of separated cells was detected by FACS-Calibur flow cytometer (Becton Dickinson, San Jose, CA, USA).

\section{Measurement of intracellular reactive oxygen species}

Production of intracellular reactive oxygen species (ROS) was measured by using the fluorescent dye 2, 7-dichlorofluorescein diacetate (DCFH-DA) as a substrate. Briefly, after indicated treatments, cells were stained with $10 \mu \mathrm{M}$ DCFH-DA in Hank's balanced salt solution (HBSS) for $30 \mathrm{~min}$ before collecting. For quantitative assessment of ROS generation, cells were collected, suspended in PBS and analyzed by FACS-Calibur flow cytometer (Becton Dickinson, San Jose, CA, USA). For image taking, DCFH-DA loaded cells were visualized using a fluorescent microscope (Olympus, Tokyo, Japan).

\section{Transfection and RNA interference}

The lentiviral vector containing GFP-LC3B and GFP-mRFP-LC3B reporter were constructed by GeneChem (Shanghai, China) and transfection was carried out according to the manufacturer's instructions. The double-stranded siRNA duplex for Atg5 (5'-GAAGUUUGUCCUUCUGCUA-3'), Nrf2 (5'-GTAAGAAGCCAGATGTTAA-3'), CHOP (5'-GC CUGGUAUGAGGACCUGC-3') and non-targeting scramble siRNA were synthesized chemically from Ribobio (Guangzhou, China) and resuspended in RNase-free buffer to a concentration of $20 \mu \mathrm{M}$. For siRNA transfection, cells were seeded into 6-well 
plates in antibiotic-free media and transfected at $30-50 \%$ confluency with $50 \mathrm{nM}$ siRNA in Opti-MEM (Invitrogen) using Lipofectamine 2000 (Invitrogen) according to the manufacturer's instructions.

\section{Statistical analysis}

Data are presented as the mean \pm SD from at least three separate experiments unless otherwise indicated. Levels of significance were evaluated by 2-tailed Student's $t$-test using GraphPad Prism 5 software (GraphPad Software Inc., La Jolla, CA, USA). A difference was considered statistically significant when $\mathrm{P}<0.05$.

\section{Abbreviations}

Atg, autophagy-related; CQ, chloroquine; CHOP, C/EBP homologous protein; CsA, cyclosporin A; DCFH-DA, 2', 7'-dichlorofluorescin diacetate; ER, endoplasmic reticulum; ERAD, ER-associated ubiquitin-proteasome degradation; GFP, green fluorescent protein; HO-1, heme oxygenase-1; LC3B, microtubule-associated protein 1 light chain 3 beta; NAC, N-acetyl-L-cysteine; NQO1, NAD $(\mathrm{P}) \mathrm{H}$ quinone oxidoreductase 1; Nrf2, NF-E2-related factor 2; PC, pancreatic cancer; ROS, reactive oxygen species; RFP, red fluorescent protein; TM, tunicamycin; TUDCA, tauroursodeoxycholic acid; UPR, unfolded protein response.

\section{Supplementary Material}

Supplementary materials and methods, figures. http://www.ijbs.com/v14p1291s1.pdf

\section{Acknowledgments}

We thank James P. Mahaffey, PhD, from Liwen Bianji, Edanz Editing China (www.liwenbianji.cn/ac), for editing the English text of a draft of this manuscript. This study was funded by The National Natural Science Foundation of China (No. 81502633 to Xu Li, No. 81772950 to Renyi Qin, No. 81602475 to Xingjun Guo, No. 81773160 to Min Wang, No. 81572429 to Jianxin Jiang).

\section{Competing Interests}

The authors have declared that no competing interest exists.

\section{References}

1. Wolfgang CL, Herman JM, Laheru DA, Klein AP, Erdek MA, Fishman EK, et al. Recent progress in pancreatic cancer. CA Cancer J Clin. 2013; 63: 318-48.

2. Michl P, Gress TM. Current concepts and novel targets in advanced pancreatic cancer. Gut. 2013:62:317-26.

3. Soave CL, Guerin T, Liu J, Dou QP. Targeting the ubiquitin-proteasome system for cancer treatment: discovering novel inhibitors from nature and drug repurposing. Cancer Metastasis Rev. 2017; 36: 717-736.

4. Russo A, Bronte G, Fulfaro F, Cicero G, Adamo V, Gebbia N, et al. Bortezomib: a new pro-apoptotic agent in cancer treatment. Curr Cancer Drug Targets. 2010; $10 \cdot 55-67$.
5. Cao B, Li J, Mao X. Dissecting bortezomib: development, application, adverse effects and future direction. Curr Pharm Des. 2013; 19: 3190-200.

6. Obeng EA, Carlson LM, Gutman DM, Harrington WJ Jr, Lee KP, Boise LH. Proteasome inhibitors induce a terminal unfolded protein response in multiple myeloma cells. Blood. 2006; 107: 4907-16.

7. Huang Z, Wu Y, Zhou X, Xu J, Zhu W, Shu Y, et al. Efficacy of therapy with bortezomib in solid tumors: a review based on 32 clinical trials. Future Oncol. 2014; 10: 1795-807.

8. Manasanch EE, Orlowski RZ. Proteasome inhibitors in cancer therapy. Nat Rev Clin Oncol. 2017; 14: 417-433.

9. Ron D, Walter P. Signal integration in the endoplasmic reticulum unfolded protein response. Nat Rev Mol Cell Biol. 2007; 8: 519-29.

10. Boyce M, Yuan J. Cellular response to endoplasmic reticulum stress: a matter of life or death. Cell Death Differ. 2006; 13: 363-73.

11. Tabas I, Ron D. Integrating the mechanisms of apoptosis induced by endoplasmic reticulum stress. Nat Cell Biol. 2011; 13: 184-90.

12. Fujita E, Kouroku Y, Isoai A, Kumagai H, Misutani A, Matsuda C, et al. Two endoplasmic reticulum-associated degradation (ERAD) systems for the novel variant of the mutant dysferlin: ubiquitin/proteasome ERAD (I) and autophagy/lysosome ERAD (II). Hum Mol Genet. 2007; 16: 618-29.

13. Ding WX, Ni HM, Gao W, Yoshimori T, Stolz DB, Ron D, et al. Linking of autophagy to ubiquitin-proteasome system is important for the regulation of endoplasmic reticulum stress and cell viability. Am J Pathol. 2007; 171: 513-24.

14. Ravikumar B, Sarkar S, Davies JE, Futter M, Garcia-Arencibia M, Green-Thompson ZW, et al. Regulation of mammalian autophagy in physiology and pathophysiology. Physiol Rev. 2010; 90: 1383-435.

15. Mathew R, Karantza-Wadsworth V, White E. Role of autophagy in cancer. Nat Rev Cancer. 2007; 7: 961-7.

16. Hashimoto D, Bläuer M, Hirota M, Ikonen NH, Sand J, Laukkarinen J. Autophagy is needed for the growth of pancreatic adenocarcinoma and has a cytoprotective effect against anticancer drugs. Eur J Cancer. 2014; 50: 1382-90.

17. Min $\mathrm{H}, \mathrm{Xu} \mathrm{M}$, Chen $\mathrm{ZR}$, Zhou JD, Huang $\mathrm{M}$, Zheng $\mathrm{K}$, et al. Bortezomib induces protective autophagy through AMP-activated protein kinase activation in cultured pancreatic and colorectal cancer cells. Cancer Chemother Pharmacol. 2014; 74: 167-76.

18. Nogueira V, Hay N. Molecular pathways: reactive oxygen species homeostasis in cancer cells and implications for cancer therapy. Clin Cancer Res. 2013; 19: 4309-14.

19. Kardeh S, Ashkani-Esfahani S, Alizadeh AM. Paradoxical action of reactive oxygen species in creation and therapy of cancer. Eur J Pharmacol. 2014; 735: 150-68.

20. Zeeshan HM, Lee GH, Kim HR, Chae HJ. Endoplasmic Reticulum Stress and Associated ROS. Int J Mol Sci. 2016; 17: 327.

21. Galadari S, Rahman A, Pallichankandy S, Thayyullathil F. Reactive oxygen species and cancer paradox: To promote or to suppress? Free Radic Biol Med. 2017; 104: 144-164.

22. Panda PK, Mukhopadhyay S, Das DN, Sinha N, Naik PP, Bhutia SK. Mechanism of autophagic regulation in carcinogenesis and cancer therapeutics. Semin Cell Dev Biol. 2015; 39: 43-55.

23. Kobayashi M, Yamamoto M. Molecular mechanisms activating the Nrf2-Keap1 pathway of antioxidant gene regulation. Antioxid Redox Signal. 2005; 7: 385-94.

24. Ma Q. Role of nrf2 in oxidative stress and toxicity. Annu Rev Pharmacol Toxicol. 2013; 53: 401-26.

25. Sporn MB, Liby KT. NRF2 and cancer: the good, the bad and the importance of context. Nat Rev Cancer. 2012; 12: 564-71.

26. Jaramillo MC, Zhang DD. The emerging role of the Nrf2-Keap1 signaling pathway in cancer. Genes Dev. 2013; 27: 2179-91.

27. Kroemer G, Galluzzi L, Brenner C. Mitochondrial membrane permeabilization in cell death. Physiol Rev. 2007; 87: 99-163.

28. Tait SW, Green DR. Mitochondria and cell death: outer membrane permeabilization and beyond. Nat Rev Mol Cell Biol. 2010; 11: 621-32.

29. Wu WK, Sakamoto KM, Milani M, Aldana-Masankgay G, Fan D, Wu K, et al. Macroautophagy modulates cellular response to proteasome inhibitors in cancer therapy. Drug Resist Updat. 2010; 13: 87-92.

30. Klionsky DJ, Abdelmohsen $\mathrm{K}$, Abe A, Abedin MJ, Abeliovich $\mathrm{H}$, Acevedo Arozena A, et al. Guidelines for the use and interpretation of assays for monitoring autophagy (3rd edition). Autophagy. 2016; 12: 1-222.

31. Kitzler TM, Papillon J, Guillemette J, Wing SS, Cybulsky AV. Complement modulates the function of the ubiquitin-proteasome system and endoplasmic reticulum-associated degradation in glomerular epithelial cells. Biochim Biophys Acta. 2012; 1823: 1007-16.

32. Qin L, Wang Z, Tao L, Wang Y. ER stress negatively regulates AKT/TSC/mTOR pathway to enhance autophagy. Autophagy. 2010; 6: $239-47$.

33. Shimodaira Y, Takahashi S, Kinouchi Y, Endo K, Shiga H, Kakuta Y, et al. Modulation of endoplasmic reticulum (ER) stress-induced autophagy by $\mathrm{C} / \mathrm{EBP}$ homologous protein (CHOP) and inositol-requiring enzyme 1a (IRE1a) in human colon cancer cells. Biochem Biophys Res Commun. 2014; 445: 524-33.

34. Tan HK, Muhammad TST, Tan ML. 14-Deoxy-11,12-didehydroandrographolide induces DDIT3-dependent endoplasmic reticulum stress-mediated autophagy in T-47D breast carcinoma cells. Toxicol Appl Pharmacol. 2016; 300: 55-69. 
35. Deniaud A, Sharaf el dein O, Maillier E, Poncet D, Kroemer G, Lemaire C, et al. Endoplasmic reticulum stress induces calcium-dependent permeability transition, mitochondrial outer membrane permeabilization and apoptosis. Oncogene. 2008; 27: 285-99.

36. Ling YH, Liebes L, Zou Y, Perez-Soler R. Reactive oxygen species generation and mitochondrial dysfunction in the apoptotic response to Bortezomib, a novel proteasome inhibitor, in human $\mathrm{H} 460$ non-small cell lung cancer cells. J Biol Chem. 2003; 278: 33714-23.

37. Selimovic D, Porzig BB, El-Khattouti A, Badura HE, Ahmad M, Ghanjati F, et al. Bortezomib/proteasome inhibitor triggers both apoptosis and autophagy-dependent pathways in melanoma cells. Cell Signal. 2013; 25: 308-18.

38. Hambright HG, Ghosh R. Autophagy: In the cROSshairs of cancer. Biochem Pharmacol. 2017; 126: 13-22.

39. Harris $\mathrm{MH}$, Thompson $\mathrm{CB}$. The role of the Bcl-2 family in the regulation of outer mitochondrial membrane permeability. Cell Death Differ. 2000; 7: 1182-91.

40. Gillies LA, Kuwana T. Apoptosis regulation at the mitochondrial outer membrane. J Cell Biochem. 2014; 115: 632-40.

41. Zhang HY, Du ZX, Meng X, Zong ZH, Wang HQ. Beclin 1 enhances proteasome inhibition-mediated cytotoxicity of thyroid cancer cells in macroautophagy-independent manner. J Clin Endocrinol Metab. 2013; 98: E217-26.

42. Chen N, Wu L, Yuan H, Wang J. ROS/Autophagy/Nrf2 Pathway Mediated Low-Dose Radiation Induced Radio-Resistance in Human Lung Adenocarcinoma A549 Cell. Int J Biol Sci. 2015; 11: 833-44. 